

\title{
Antioxidant Study of Three Curcuma Species and Screening of Antioxidant Components Using Stepwise Regression Analysis
}

\author{
Shu-Ping Hu, YuAn Zhang and Zheng XIANG*
}

School of Pharmaceutical Sciences, Wenzhou Medical University, Wenzhou, Zhejiang 325035, P.R. China

*Corresponding author: Tel/Fax: +86 577 86699156; E-mail: xzh0077@126.com

\begin{abstract}
This study evaluated the effectiveness of the antioxidant properties of fresh and dry rhizomes of Curcuma phaeocaulis, C. kwangsiensis and $C$. wenyujin based on their 1,1-diphenyl-2-picrylhydrazyl and hydroxyl radical scavenging activities, ferrous ion chelating ability and reducing power. Stepwise regression analysis was used to establish the component-effect relationship between HPLC profiling and halfmaximal effective concentrations. The results demonstrated that $95 \%$ ethanol and ethyl acetate extracts from the three Curcuma species at lower concentrations have higher DPPH and $\mathrm{OH}$ free radical scavenging rates. The leave-one-out cross-validation coefficients of the component-effect model of DPPH, OH and reduction methods were greater than 0.8 , indicating that the component-effect model is highly robust and predictive. This study suggests that $95 \%$ ethanol and ethyl acetate extracts from three Curcumas are rich sources of antioxidant substances and that multivariate regression analysis can be a useful tool for screening antioxidant components.
\end{abstract}

Keywords: Curcuma, Stepwise regression, Antioxidant, Multivariate regression analysis.

\section{INTRODUCTION}

Curcuma phaeocaulis, Curcuma kwangsiensis and Curcuma wenyujin belong to the Zingiberaceae family, which are distributed widely throughout the tropics and subtropics, particularly in China. Their roots, called "Ezhu," are more commonly used as Chinese herbal medicines and food additives and their annual market consumption exceeds 20,000 tons of dried herbs. Pharmacological studies have found that they have antitumor $^{1-4}$, antiinflammatory ${ }^{5-7}$, antibacterial and antiviral ${ }^{8,9}$ effects, among others and inhibit lipid peroxidation ${ }^{10}$. Free radicals (FRs) and reactive oxygen species (ROS) produced during metabolism are major contributors to various chronic diseases. Increasing evidence that oxidative damage plays an important role in the development of chronic, age-related degenerative diseases supports this point ${ }^{11}$. Antioxidants delay or prevent the oxidation of free radicals and/or reactive oxygen species and thus reduce the risk of disease ${ }^{12}$. The secondary metabolites from curcuma plants mainly contain sesquiterpenoids, including $\beta$-elemene, curcumol, germacrone, curdione and neocurdione, as well as curcuminoids, including curcumin, demethoxy-curcumin and bisdemethoxy-curcumin ${ }^{13-18}$. Data suggesting that polyphenolic curcuminoids and some sesquiterpenoid antioxidants may be useful for the prevention and/or treatment of some age-related degenerative diseases have been reported $^{19}$. The present study thus endeavored to evaluate the antioxidant properties of the above mentioned Curcuma species, with the aim of exploring more sources of natural antioxidants and thereby promoting their development.

Screening of antioxidant components from medicinal plants is complicated: Monomers are usually the first to be isolated and their antioxidant capacities are then assayed individually. However, this screening way is time-consuming and uneconomical. In addition, because a synergistic action exists among antioxidant components, research has shown that several antioxidant components at lower concentrations have better antioxidant capacity, which contributes to their safe use as food additives ${ }^{20}$. These data render knowledge on how antioxidant components or clusters can be more effectively screened very crucial. Multiple regression analysis can establish the relationship between a dependent variable and independent variables and stepwise regression is commonly used multiple regression approach ${ }^{21}$. In the regression process, independent variables are individually introduced into the regression equation according to the number and order of their partial correlation coefficients. The partial correlation coefficients of each introduced variable are then statistically tested; significant variables remain in the regression equation, whereas no significant ones are deleted so that other variables may be selected. Given that new variables are introduced into the equation, some original variables are not likely to be statistically significant due to their interactions and should be removed 
from the equation based on statistical tests; only significant variables are thus retained for regression analysis. The optimal regression equation is obtained once the introduction and elimination of variables stop. As antioxidant capacity was regarded as the dependent variable and peak areas of HPLC chromatographic profilings of Curcuma extracts were taken as independent variables in this study. Significant antioxidant substances were selected as variables in the regression model.

The primary objective of this study was to systematically evaluate the antioxidant capacities of different solvent extracts from fresh and dried herbs of $C$. phaeocaulis, $C$. kwangsiensis and $C$. wenyujin according to their 1,1-diphenyl-2-picrylhydrazyl (diphenyl-hydrazine bitter; $\left.\mathrm{DPPH}^{\circ}\right)$ and hydroxyl radical $\left(\mathrm{OH}^{\circ}\right)$ scavenging activities, ferrous ion $\left(\mathrm{Fe}^{2+}\right)$ chelating ability and ferric ion reducing power. Multivariate stepwise regression was used to establish component-effect model and to screen antioxidant substances or clusters from curcuma plants.

\section{EXPERIMENTAL}

Ferrous triazine, deoxyribose and DPPH were obtained from Sigma Chemical Co. (St. Louis, MO, USA). $\mathrm{FeSO}_{4} \cdot 7 \mathrm{H}_{2} \mathrm{O}$, potassium ferricyanide, disodium hydrogen phosphate, sodium dihydrogen phosphate, trichloroacetic acid, ferric chloride, $\mathrm{H}_{2} \mathrm{O}_{2}$ and thiobarbituric acid were purchased from Guoyao Company (China). Folin-Ciocalteu reagent, butylated hydroxyanisole and gallic acid were obtained from the China Drugs and Biological Products Inspection Institute (purity, $\geq 98 \%$ ). All reagents were of analytical grade.

Sample preparation: Samples of $C$. phaeocaulis, $C$. kwangsiensis and $C$. wenyujin were collected before and after January 2012 from their respective areas of original cultivation in China: Wenjiang, Sichuan Province; Lingshan, Guangxi Province; and Ruian, Zhejiang Province. They were identified by Dong Jian-yong (Pharmacy Department, Wenzhou Medical College, Wenzhou, Zhejiang, China). The dry herbs were processed by boiling the fresh samples for $40 \mathrm{~min}$, followed by parching at $35^{\circ} \mathrm{C}$ until a constant weight was obtained. The samples to be tested were cut into smaller pieces, ground into powder and then passed through a 20-mesh $(0.9 \mathrm{~mm})$ sieve. Twenty-five grams of the fresh and dried powder samples was placed in a Soxhlet apparatus and extracted for $6 \mathrm{~h}$. One hundred milliliters of solvent containing distilled water, $95 \%$ ethanol and ethyl acetate was used as an extractant. Extracts were cooled to room temperature and then concentrated to 10 $\mathrm{mL}$ using a vacuum rotary evaporator (Büchi R-210, Flawil, Switzerland). Each concentrated extract was serially diluted to approximately $50 \%$ until eight samples of varying concentrations were available for use in the assays. Concentrations ranged from approximately 40 to $0.05 \mathrm{mg} / \mathrm{mL}$ for both fresh and dried extracts. All concentrations were calculated on a dry rhizome mass basis. Extracts were stored in the dark at $-5^{\circ} \mathrm{C}$ prior to use.

DPPH free radical scavenging: The capacity of extracts to remove DPPH was determined using a modified version of the method described by Shimada et al. ${ }^{22}$. Briefly, $2 \mathrm{~mL}$ of extracts and $1 \mathrm{~mL}$ of freshly prepared $0.2 \mathrm{mM}$ DPPH methanolic solution were thoroughly mixed and kept in the dark for $0.5 \mathrm{~h}$. The absorbance of the reaction mixture at $517 \mathrm{~nm}$ was measured using a spectrophotometer. Each sample was detected three times and expressed as mean \pm SD. The percentage of free radical scavenging activity was calculated as follows:

$$
\text { Scavenging activity }(\%)=\left(1-\frac{A_{\text {Extract }}}{A_{\text {Control }}}\right) \times 100
$$

where $\mathrm{A}_{\text {Control }}$ is the absorbance of the blank sample and $\mathrm{A}_{\text {Extract }}$ is the absorbance of test samples.

$\mathrm{OH}^{\bullet}$ radical scavenging effect: $\mathrm{OH}^{\bullet}$ radical scavenging effect was determined as previously described ${ }^{23}$. Reaction mixtures contained $\mathrm{FeSO}_{4}(0.2 \mathrm{~mL}, 3 \mathrm{mM})$, EDTA $(0.2 \mathrm{~mL}$, $3 \mathrm{mM}), \mathrm{H}_{2} \mathrm{O}_{2}(0.2 \mathrm{~mL}, 3 \mathrm{mM})$, deoxyribose $(0.5 \mathrm{~mL}, 5 \mathrm{mM})$ and various concentrations of extracts $(1.5 \mathrm{~mL})$. All mixtures were dissolved in $\mathrm{KH}_{2} \mathrm{PO}_{4} / \mathrm{KOH}$ buffer $(20 \mathrm{mM}$, pH 7.4). After incubation at $37^{\circ} \mathrm{C}$ for $1 \mathrm{~h}$, trichloroacetic acid $(1 \mathrm{~mL}, 2.8 \%$ $\mathrm{w} / \mathrm{v})$ and thiobarbituric acid $(1 \mathrm{~mL}, 1 \% \mathrm{w} / \mathrm{v})$ were added and the mixture was heated in a water bath at $100{ }^{\circ} \mathrm{C}$ for 15 min. The absorbance of the resulting solution at $532 \mathrm{~nm}$ was measured. $\mathrm{OH}^{\bullet}$ scavenging activity was calibrated, with a higher percentage indicating higher antioxidant activity.

$\mathrm{Fe}^{2+}$ chelating effect: This study adopted the $\mathrm{Fe}^{2+}$ chelating assay used by Singh and Rajini ${ }^{24}$. FeSO 4 (0.1 mL, $\left.2 \mathrm{mM}\right)$ was mixed with various concentrations of extracts $(1 \mathrm{~mL})$, followed by ferrozine $(0.1 \mathrm{~mL}, 5 \mathrm{mM})$. Absorbance at $562 \mathrm{~nm}$ was measured after $10 \mathrm{~min}$. The ability of extracts to chelate ferrous ions was calculated as follows:

$$
\text { Chelating effect }(\%)=\left(1-\frac{A_{\text {Extract }}}{A_{\text {Control }}}\right) \times 100
$$

where $\mathrm{A}_{\text {Extract }}$ and $\mathrm{A}_{\text {Control }}$ are the absorbance of the extract and negative control, respectively.

Reducing power test: Reducing power was measured according to the method described by Duh and Yen ${ }^{25}$. Various concentrations of extracts $(2.5 \mathrm{~mL})$, phosphate buffer $(200$ $\mathrm{mM}, \mathrm{pH} 6.6,2.5 \mathrm{~mL}$ ) and potassium hexacyanoferrate solution $(1 \% \mathrm{v} / \mathrm{w}, 2.5 \mathrm{~mL})$ were placed in a test tube and heated at $50{ }^{\circ} \mathrm{C}$ for $20 \mathrm{~min}$. The test tube was then cooled in an ice bath, after which trichloroacetic acid $(0.5 \mathrm{~mL}, 10 \%)$ was added. After centrifugation at $3000 \mathrm{~g}$ for $10 \mathrm{~min}$, a $5 \mathrm{~mL}$ aliquot of the supernatant was mixed and reacted with distilled water $(5 \mathrm{~mL})$ and ferric chloride $(1 \mathrm{~mL}, 0.1 \%)$ for $10 \mathrm{~min}$. Finally, absorbance at $700 \mathrm{~nm}$ was measured, with increased absorbance of the reaction mixture indicating increased reducing power.

HPLC conditions: HPLC was performed on an Agilent Series 1100 system (Agilent Technologies, USA). A Hypersil ODS column ( $250 \mathrm{~mm} \times 4 \mathrm{~mm}$ I.D., $5 \mathrm{~mm}$ ) was used for separation. The mobile phase consisted of water and acetonitrile with gradient elution as follows: $0-15 \mathrm{~min}, 30-45 \%$ acetonitrile; 30-40 min, 47-60 \% acetonitrile; 40-50 min, 60-90\% acetonitrile; $50-60 \mathrm{~min}, 90-100 \%$ acetonitrile. The flow rate was $1 \mathrm{~mL} / \mathrm{min}$. The samples were detected at $210 \mathrm{~nm}$. The column temperature was maintained at $30^{\circ} \mathrm{C}$ and each sample was tested three times.

Data processing and statistical analysis: A multivariate statistical method of stepwise regression was used for screening active components in extracts to build a model of antioxidant components and antioxidant capacity. Stepwise regression was 
directly performed using Matlab 7.0 procedures. Chromatographic peaks of extracts with a limit of quantification (signalto-noise ratio) $>10$ were labeled and integrated. The chromatographic peak areas and retention times were then obtained, removing $70 \%$ missing peaks at the same retention times. The 20 chromatographic peaks, which constituted an $18 \times 20$ matrix, are shown in Fig. 1 . These variable data were normalized and used for stepwise regression analysis. The dependent variable was represented by $\mathrm{EC}_{50}$, which is the sample concentration in which half of the radicals were removed. In addition, the experimental results were expressed as the mean \pm SD of three replicates. All scavenging and chelating curves were generated by GraphPad Prism software.

\section{RESULTS AND DISCUSSION}

HPLC profilings of extracts: A $1 \mathrm{~mL}$ supernatant of the half-maximal effective concentration $\left(\mathrm{EC}_{50}\right)$ was filtered through a $0.45 \mu \mathrm{m}$ membrane and the filtrate $(20 \mu \mathrm{L})$ was detected for HPLC analysis. HPLC chromatogram of mix samples from three fresh or dried curcumas are shown in Fig. 1.

As shown in Fig. 2, the HPLC profilings of extracts from different solvents and species significantly differed, but those from the same species of dried and fresh herbs were similar. In the ethyl acetate extracts, the contents of peaks 8, 9, 10 and 17 (components) in C. phaeocaulis were higher than those in $C$. wenyujin and $C$. kwangsiensis, especially for peak 17 . The contents of peaks $8,9,10,14,19$ and 20 in $C$. wenyujin were relatively high, whereas the components of peak 14 in $C$. phaeocaulis and C. kwangsiensis were particularly small. The



contents of peaks 8, 9, 10 and 15 in $C$. kwangsiensis were higher than those in $C$. phaeocaulis and $C$. wenyujin, with the components of peak 15 detected in considerably small amounts. The HPLC chromatograms of $95 \%$ ethanol and ethyl acetate extracts from the three Curcuma species were similar. On the other hand, the HPLC chromatograms of water extracts showed few components, among which peaks 9 and 10 were the main ingredients, although their contents were low.

$\mathrm{DPPH}^{\bullet}$ radical scavenging activity: The $\mathrm{DPPH}^{\bullet}$ radical is a stable nitrogen-centered free radical widely used for screening the in vitro antioxidant activities of fruits and vegetables, plant extracts and foods ${ }^{26}$. The DPPH radical scavenging rates of the water, $95 \%$ ethanol and ethyl acetate extracts from C. phaeocaulis, C. kwangsiensis and $C$. wenyujin are shown in Fig. 3a,b. The clearance rates of ethyl acetate extracts
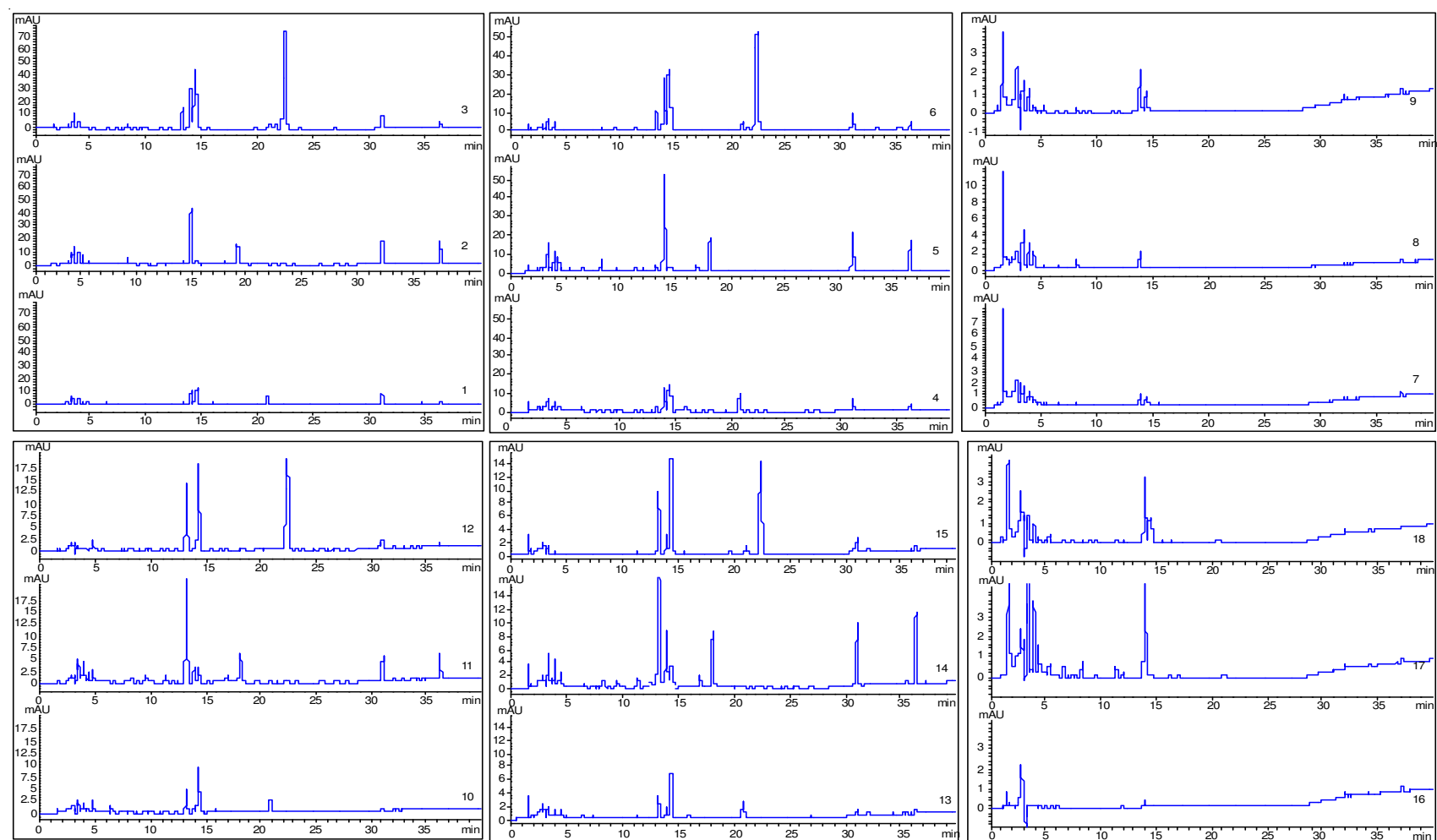

Fig. 2. HPLC chromatograms of the 18 samples: 1, ethyl acetate (EAc) extracts from C. kwangsiensis; 2, EAc extracts from C. wenyujin; 3 , EAc extracts from $C$. phaeocaulis; 4, $95 \%$ ethanol (EtOH) extracts from C. kwangsiensis; 5, $95 \%$ EtOH extracts from C. wenyujin; 6, $95 \%$ EtOH extracts from C. phaeocaulis; $7, \mathrm{H}_{2} \mathrm{O}$ extracts from C. kwangsiensis; $8, \mathrm{H}_{2} \mathrm{O}$ extracts from C. wenyujin; $9, \mathrm{H}_{2} \mathrm{O}$ extracts from $C$. phaeocaulis; 10 , EAc extracts from fresh $C$. kwangsiensis; 11, EAc extracts from fresh $C$. wenyujin; 12, fresh EAc extracts from fresh C. phaeocaulis; $13,95 \%$ EtOH extracts from fresh C. kwangsiensis; $14,95 \% \mathrm{EtOH}$ extracts from fresh $C$. wenyujin; $15,95 \% \mathrm{EtOH}$ extracts from fresh $C$. phaeocaulis; $16, \mathrm{H}_{2} \mathrm{O}$ extracts from fresh $C$. kwangsiensis; $17, \mathrm{H}_{2} \mathrm{O}$ extracts from $C$. wenyujin; $18, \mathrm{H}_{2} \mathrm{O}$ extracts from fresh $C$. phaeocaulis. 
approximated those of dried $95 \%$ ethanol extracts at the tested concentrations and significant differences in clearance among different species were not observed. The water extracts exhibited lower clearance rates compared with the ethyl acetate and $95 \%$ ethanol extracts. The antioxidant capacities of different solvent extracts in the range of $0-10 \mathrm{mg} / \mathrm{mL}$ clearly showed concentration dependence.

For the fresh extracts, the antioxidant capacities of different solvent extracts in the range of $0-25 \mathrm{mg} / \mathrm{mL}$ also demonstrated concentration dependence. At lower concentrations (0-10 $\mathrm{mg} / \mathrm{mL}$ ), the ethyl acetate and $95 \%$ ethanol extracts from $C$. wenyujin had higher clearance rates compared with those from the two other species. In addition, the scavenging rates of fresh extracts with the same solvent differed. For the same Curcuma species, the clearance rates were in the following order: $95 \%$ ethanol extracts $>$ ethyl acetate extracts $>$ water extracts.

Stepwise regression was used in selecting active components against the DPPH radical to define the component-effect relationship between DPPH scavenging rate and antioxidant components. The following regression model was used: $\mathrm{Y}=$ $85.26 \cdot X_{14}+29.50 \cdot X_{15}+799.00 \cdot X_{16}$, where $Y$ represents the $\mathrm{EC}_{50}$ of DPPH and $\mathrm{X}_{\mathrm{i}}$ is the ith peak (component) in chroma- tograms. The correlation coefficient of the model was 0.854 and the leave-one-out cross-validation coefficient was 0.823 .

$\mathrm{OH}^{\bullet}$ radical scavenging activity: The $\mathrm{OH}^{\bullet}$ radical is reactive oxygen species with high reactivity capable of attacking most biological substrates, such as DNA, polyunsaturated fatty acids and proteins. The prevention of such deleterious reactions is highly significant in terms of both human health and the shelf-life of foods, cosmetics and pharmaceutical products $^{27,28}$. The $\mathrm{OH}^{\bullet}$ radical clearance rates of $C$. phaeocaulis, C. kwangsiensis and C. wenyujin are shown in Fig. 3c,d. Both dried and fresh herbs with ethyl acetate and $95 \%$ ethanol extracts at a lower concentration $(0.01 \mathrm{mg} / \mathrm{mL})$ showed higher $\mathrm{OH}^{\bullet}$ clearance rates, suggesting that some chemical components in these Curcuma species have strong $\mathrm{OH}^{\bullet}$ radical scavenging capacity. Concentration-dependent (concentration range, $0-0.05 \mathrm{mg} / \mathrm{mL}$ ) inhibition against $\mathrm{OH}^{\bullet}$ radicals was observed in all assays. The clearance rates of water extracts were significantly lower than those of ethyl acetate and $95 \%$ ethanol extracts, suggesting that antioxidant components should be mainly medium and weakly polar components. The following stepwise regression model for $\mathrm{OH}^{\bullet}$ clearance rate was used: $\mathrm{Y}=73.02 \cdot \mathrm{X}_{19}-203.84 \cdot \mathrm{X}_{2}+38.98 \cdot \mathrm{X}_{9}+89.21$, with a correlation

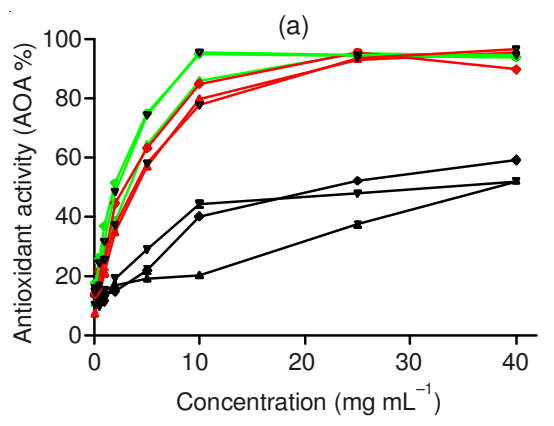

(d)
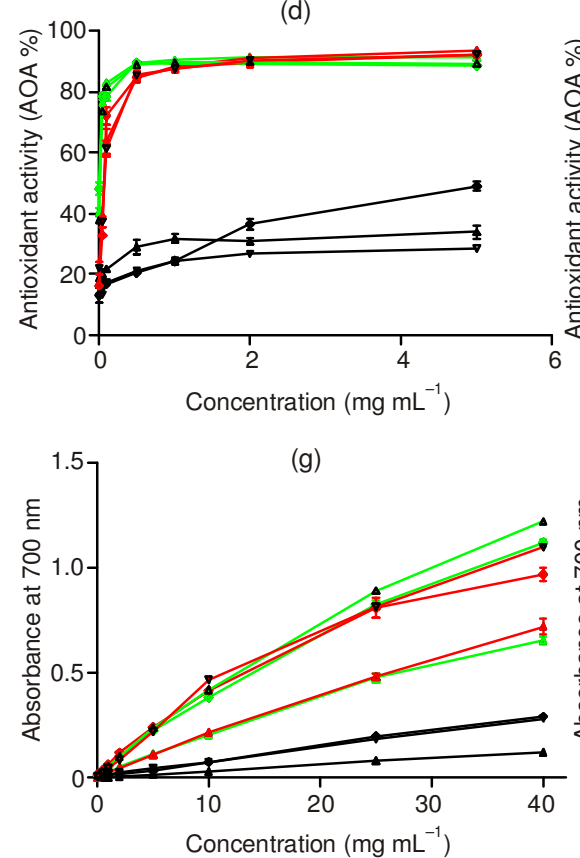

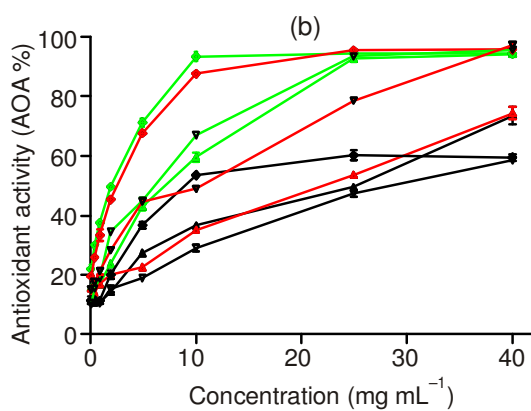

(e)

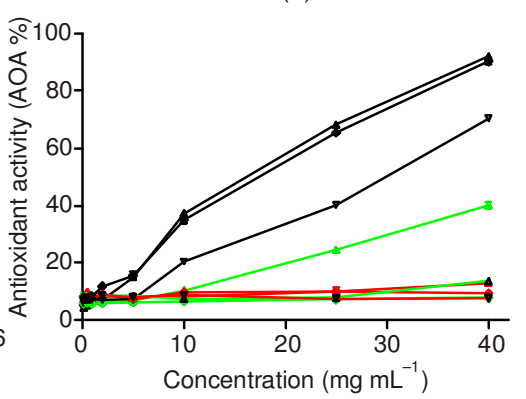

(h)

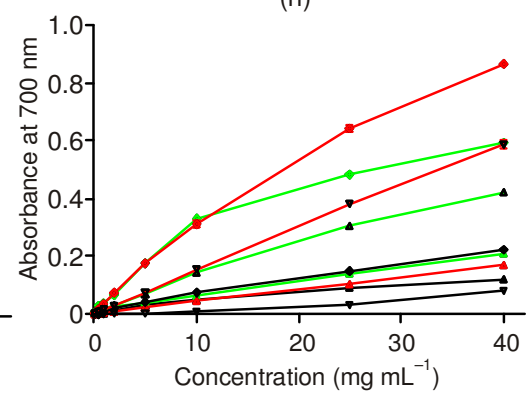

(c)

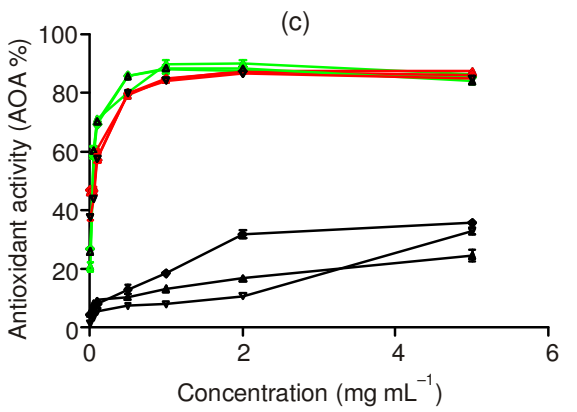

(f)

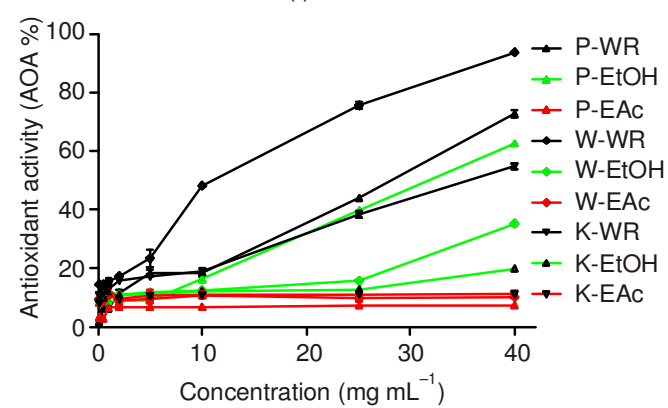

(i)

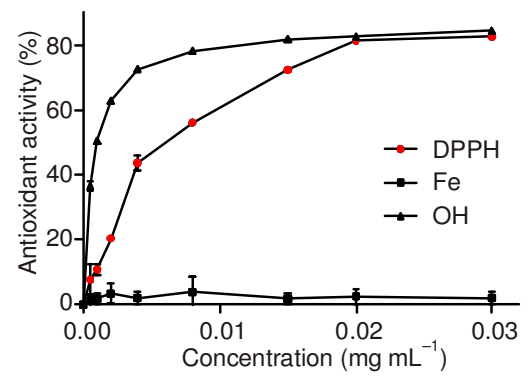

Fig. 3. Evaluation of antioxidant capacity of extracts from C. phaeocaulis, C. kwangsiensis and C. wenyujin and curcumin (I). The dried (a) and fresh (b) against DPPH; The dried (c) and fresh (d) against OH radicals; The dried (e) and fresh (f) against chelating effects; The dried (g) and fresh (h) against ferric reducing powers. P-WR: $\mathrm{H}_{2} \mathrm{O}$ extracts from $C$. phaeocaulis; P-EtOH: EtOH extracts from C. phaeocaulis; P-EAc: EAc extracts from C. phaeocaulis; W-WR: $\mathrm{H}_{2} \mathrm{O}$ extracts from $C$. wenyujin; W-EtOH: EtOH extracts from $C$. wenyujin; W-EAc: EAc extracts from $C$. wenyujin; K-WR: $\mathrm{H}_{2} \mathrm{O}$ extracts from C. kwangsiensis; K-EtOH: EtOH extracts from C. kwangsiensis; K-EAc: EAc extracts from C. kwangsiensis. 
coefficient of 0.903 and a leave-one-out cross-validation coefficient of 0.882 , which may indicate components 9 (curcumin) and 19 (unknown composition) have better $\mathrm{OH}$ scavenging rates.

$\mathbf{F e}^{2+}$ chelating effect: Iron with high reaction activity is regarded as an important accelerator of lipid oxidation, indicating that chelating $\mathrm{Fe}^{2+}$ can protect against oxidative damage $^{29}$. The chelating effect of water and $95 \%$ ethanol extracts on $\mathrm{Fe}^{2+}$ increased as concentrations increased from 0.1 to $40 \mathrm{mg} / \mathrm{mL}$ (Fig. 3e,f). The chelating effect of water extracts was higher than that of $95 \%$ ethanol extracts, whereas ethyl acetate extracts had almost no chelating effect on $\mathrm{Fe}^{2+}$. As shown in Fig. 3e,f, compared with the $\mathrm{OH}^{\bullet}$ radical scavenging effect, the chelating effect was not effective for $C$. phaeocaulis, $C$. kwangsiensis and $C$. wenyujin. For the fresh extracts, at $25 \mathrm{mg} / \mathrm{mL}$, the chelating effects were in the descending order of $C$. wenyujin $>C$. phaeocaulis $>C$. kwangsiensis. The following stepwise regression model for ferrous chelation rate was used: $\mathrm{Y}=231.66 \cdot \mathrm{X}_{16}+13.95$, with a correlation coefficient of 0.388 and a leave-one-out cross-validation coefficient of 0.35 .

Reducing power: Reducing power was measured according to the method described by Duh and Yen ${ }^{25}$. Fig. $3(\mathrm{~g}, \mathrm{~h})$ shows the reducing powers of different solvent extracts from C. phaeocaulis, $C$. kwangsiensis and $C$. wenyujin. The greater absorbance was, the stronger reducing power was and the better antioxidant capacity was. The reducing powers of different solvent extracts increased as concentration increased. The reducing power of $95 \%$ ethanol extracts approximated that of ethyl acetate extracts and the reducing power of water extracts was significantly lower compared with that of the two other extracts. Water extracts exhibited marginal reducing power for the dried samples in the range of $0.1-40 \mathrm{mg} / \mathrm{mL}$. At $25 \mathrm{mg} / \mathrm{mL}$, the reducing powers of the three Curcuma species were in the descending order of $C$. wenyujin $>C$. kwangsiensis $>C$. phaeocaulis. The following stepwise regression model for reduction absorption was used: $\mathrm{Y}=2.54 \cdot \mathrm{X}_{16}+0.36 \cdot \mathrm{X}_{19}$ $0.053 \cdot X_{17}+0.035$, with a correlation coefficient of 0.905 and a leave-one-out cross-validation coefficient of 0.885 .

Fig. 2 showed the HPLC chromatograms of the chemical components and contents of different extracts varied, which represents the material basis for their different antioxidant capacities. Therefore, $\mathrm{EC}_{50}$ and corresponding concentrations of extracts were used as the dependent variable and independent variables, respectively, to build a multivariate statistical regression model for screening antioxidant components. The stepwise regression model of the 18 samples, except for the ferrous chelation model, indicated that the three other antioxidation methods can build a better regression equation model with a correlation coefficient greater than 0.85 and a leaveone-out cross-validation correlation coefficient greater than 0.8 , suggesting that the regression model has good robustness and predictive ability and that a good component-effect relationship exists between active components and their antioxidant capacity. Compared with water extracts, the ethyl acetate and $95 \%$ ethanol extracts from C. phaeocaulis, C. kwangsiensis and $C$. wenyujin exhibited good DPPH and $\mathrm{OH}^{\bullet}$ free radical scavenging activities, suggesting that the antioxidant consti- tuents in the extracts are of high antioxidant capacity. Curcumin (shown by the HPLC retention time as component 9 was selected for investigating the screening capacity of regression models to validate antioxidant ability using the above mentioned methods. The results are shown in Fig. 3i, with curcumin at lower concentrations exhibiting a higher $\mathrm{OH}^{\bullet}$ free radical scavenging capacity, which is consistent with the regression equation. Much research has shown that curcumin is a strong active antioxidant substance ${ }^{30-32}$. Furthermore, it has almost no ferrous chelating ability and does not appear in the ferrous chelation regression model. These findings indicate that multivariate statistical regression analysis can help screen active components. Many studies have also reported that, in addition to curcumin, other ingredients from Curcuma plants, such as curcumin analogues and sesquiterpene, also have antioxidant capacity $^{33,34}$. However, due to a lack of standard substances, other potential active components, such as components 14 , 15,16 and 19, could not be verified by testing.

In the regression equations, several components were usually introduced into the regression model simultaneously, indicating that synergistic or antagonistic interactions may exist among the antioxidant components. Data suggesting a wide range of synergism and antagonism between the antioxidant components contained in foods have been reported ${ }^{20,35,36}$. Therefore, strategic selection of foods or diets to maximize synergisms and minimize antagonisms in antioxidant activity is crucial. In the DPPH experiment, the combination of components 14, 15 and 16 demonstrated good antioxidant capacity, suggesting that these three components have synergistic antioxidant ability. The $\mathrm{OH}^{\bullet}$ free radical scavenging and reduction methods suggest that components 9 and 19 as well as components 16 and 19 may have synergistic antioxidant capacity, whereas components 2 and 17 may have antagonistic effects. In the four models, components 16 and 19 were introduced into the regression equation thrice and twice, respectively, suggesting that these two components are potential antioxidant substances.

\section{Conclusion}

We systematically evaluated the antioxidant capacities of different extracts from C. phaeocaulis, C. kwangsiensis and C. wenyujin. The results showed that ethyl acetate and $95 \%$ ethanol extracts have good scavenging rates against DPPH and $\mathrm{OH}$ radicals at lower concentrations, indicating that the extracts contain some antioxidant components. Multivariate statistical analysis found that components 19, 14, 15, 16 and 19 in the HPLC chromatograms might be the main antioxidant components. The antioxidant capacity of curcumin (component 9) was further validated by several antioxidant experiments, suggesting that multivariate statistics can be used for the rapid screening of antioxidant components from medicinal plants.

\section{ACKNOWLEDGEMENTS}

This work was sponsored by Zhejiang Provincial Program for the Cultrivation of High-level Innovation Health talents, the Traditional Chinese Medicine Research Project of Zhejiang Province (2010ZB087) and the Nature Foundation Committee of Wenzhou, China (Y20070057). 


\section{REFERENCES}

1. Z.Y. Yu, R. Wang, L.Y. Xu, S.W. Xie, J.H. Dong and Y.K. Jing, PLoS ONE, 6, e15843 (2011).

2. L. Yang, D.D. Wei, Z. Chen, J.S. Wang and L.Y. Kong, Phytomedicine, 18, 710 (2011)

3. X.P. Chen, L.X. Pei, Z.F. Zhong, J.J. Guo, Q.W. Zhang and Y.T. Wang, Phytomedicine, 18, 1238 (2011)

4. Y.Q. Yao, X. Ding, Y.C. Jia, C.X. Huang, Y.Z. Wang and Y.H. Xu, Cancer Lett., 264, 127 (2008).

5. J. Zhou, F. Qu, H.J. Zhang, X.H. Zhuge and L.Z. Cheng, Afr. J. Tradit. Complement. Altern. Medicines, 7, 339 (2010).

6. K. Komatsu, K. Tanaka, Y. Kuba and A. Ina, Yakugaku Zasshi-Journal of the Pharmaceutical Society of Japan, 127, 40 (2007).

7. C. Tohda, N. Nakayama, F. Hatanaka and K. Komatsu, Evid. Based Complement. Alternat. Med., 3, 255 (2006).

8. Q. Ming, F. Sun, J.W. Liu, Z.Q. Liu, S.Q. Zhang, Y.Q. Le and S.Y. Xing, Chin. J. Gerontol., 24, 267 (2004).

9. Q. Xia, Z.G. Huang, S.P. Li, P. Zhang, J. Wang and L.N. He, Chin. Pharmacol. Bull, 20, 357 (2004).

10. C. Xu, Z. Haiyan, Z. Hua, Z. Jianhong and D. Pin, Int. J. Biol. Macromol., 44, 138 (2009).

11. A.K. Atoui, A. Mansouri, G. Boskou and P. Kefalas, Food Chem., 89, 27 (2005).

12. S. Lee, N.M. Gharavi, H. Honda, I. Chang, B. Kim, N. Jen, R. Li, A. Zimman and J.A. Berliner, Free Radic. Biol. Med., 47, S145 (2009).

13. Y. Lou, F. Zhao, H. He, K.F. Peng, L.X. Chen and F. Qiu, Chem. Biodivers., 7, 1245 (2010).

14. Y.Y. Dang, X.C. Li, Q.W. Zhang, S.P. Li and Y.T. Wang, J. Sep. Sci., 33, 1658 (2010)

15. Z.J. Ma, Z.K. Meng and P. Zhang, Fitoterapia, 80, 374 (2009).

16. Y. Lou, F. Zhao, Z.H. Wu, K.F. Peng, X.C. Wei, L.X. Chen and F. Qiu, Helv. Chim. Acta, 92, 1665 (2009).

17. W. Huang, P. Zhang, Y.C. Jin, Q. Shi, Y.Y. Cheng, H.B. Qu and Z.J. Ma, Helv. Chim. Acta, 91, 944 (2008).
18. F. Fang, Z.H. Cheng, Y.L. Guo and Y.B. Cai, Chin. J. Chem., 24, 1346 (2006).

19. G.K. Jayaprakasha, L. Jaganmohan Rao and K.K. Sakariah, Food Chem., 98, 720 (2006).

20. S. Wang, K.A. Meckling, M.F. Marcone, Y. Kakuda and R. Tsao, J. Agric. Food Chem., 59, 960 (2011).

21. D.G. Kabe, J. Am. Stat. Assoc., 58, 770 (1963).

22. K. Shimada, K. Fujikawa, K. Yahara and T. Nakamura, J. Agric. Food Chem., 40, 945 (1992).

23. M. Paya, B. Halliwell and J.R.S. Hoult, Biochem. Pharmacol., 44, 205 (1992).

24. N. Singh and P.S. Rajini, Food Chem., 85, 611 (2004).

25. P.D. Duh and G.C. Yen, Food Chem., 60, 639 (1997).

26. C. Sanchez-Moreno, Food Sci. Technol. Int., 8, 121 (2002).

27. S. Sasaki, T. Ohta and E.A. Decker, J. Agric. Food Chem., 44, 1682 (1996).

28. L.W. Aurand, N.H. Boone and G.G. Giddings, J. Dairy Sci., 60, 363 (1977).

29. J.P. Kehrer, Toxicology, 149, 43 (2000).

30. Y.J. Shang, X.L. Jin, X.L. Shang, J.J. Tang, G.Y. Liu, F. Dai, Y.P. Qian, G.J. Fan, Q. Liu and B. Zhou, Food Chem., 119, 1435 (2010).

31. S. Pinlaor, P. Yongvanit, S. Prakobwong, B. Kaewsamut, J. Khoontawad, P. Pinlaor and Y. Hiraku, Mol. Nutr. Food Res., 53, 1316 (2009).

32. A. Galano, R. Alvarez-Diduk, M.T. Ramirez-Silva, G. Alarcon-Angeles and A. Rojas-Hernandez, Chem. Phys., 363, 13 (2009).

33. M. Cousins, J. Adelberg, F. Chen and J. Rieck, Ind. Crops Prod., 25, 129 (2007)

34. S.M. Al-Reza, A. Rahman, M.A. Sattar, M.O. Rahman and H.M. Fida, Food Chem. Toxicol., 48, 1757 (2010).

35. T.L. Parker, S.A. Miller, L.E. Myers, F.E. Miguez and N.J. Engeseth, J. Agric. Food Chem., 58, 209 (2010).

36. C.S. Romano, K. Abadi, V. Repetto, A.A. Vojnov and S. Moreno, Food Chem., 115, 456 (2009). 